\title{
Patterns of neuropsychological impairment in MCI patients with small subcortical infarcts or hippocampal atrophy
}

\author{
GUIDO GAINOTTI, MONICA FERRACCIOLI, MARIA GABRIELLA VITA, \\ AND CAMILLO MARRA \\ Neuropsychology Service of the UCSC/Policlinico Gemelli, Catholic University of Rome, Rome, Italy \\ (Received September 3, 2007; Final Revision April 1, 2008; Accepted April 1, 2008)
}

\begin{abstract}
We investigated whether MCI patients with hippocampal atrophy or multiple subcortical infarcts demonstrate neuropsychological patterns and markers considered typical of Alzheimer's disease (AD) and of vascular dementia (VD), respectively. An extensive neuropsychological battery, including tests of memory, visual-spatial and executive functions, language, attention, praxis and psychomotor speed, was administered to 36 mild cognitive impairment (MCI) patients with hippocampal atrophy and $41 \mathrm{MCI}$ patients with multiple subcortical infarcts. Both groups of MCI patients were very mildly impaired and well matched in terms of MMSE scores. A clear, disproportionately severe, episodic memory disorder was observed in MCI patients with hippocampal atrophy. A less specific neuropsychological profile, consisting of impairment on an Action Naming task that is sensitive to frontal lobe lesions, was observed in MCI patients with multiple subcortical infarcts. In MCI patients, a disproportionately severe episodic memory impairment strongly points to an Alzheimer's type brain pathology, whereas the prevalence of executive deficits and other frontal lobe symptoms are a much weaker diagnostic marker of small vessel subcortical disease. (JINS, 2008, 14, 611-619.)
\end{abstract}

Keywords: Vascular cognitive impairment, Prodromal Alzheimer's disease, Episodic memory disorders, Executive functions, Action naming

\section{INTRODUCTION}

The term Mild Cognitive Impairment (MCI) is used to identify nondemented patients who have an increased risk of developing a dementia of the Alzheimer's type (DAT). The defining features of the MCI are the presence of subjective memory complaints and objective memory impairment, the absence of obvious impairment in other cognitive domains, the absence of deficits in daily living activities, and an absence of dementia (Dubois \& Albert, 2004; Petersen et al., 1999; Ritchie \& Touchon, 2000). Studies conducted to date have shown that MCI patients are at increased risk of developing DAT, although the nature of this risk is very different

Correspondence and reprint requests to: Guido Gainotti, Neuropsychology Service, Policlinico Gemelli, Catholic University of Rome, Largo A. Gemelli, 8, 00168 Roma, Italy. E-mail: gainotti@rm.unicatt.it from study to study as a function of clinical, neuroradiological, and neuropsychological variables (e.g., Nordlund et al., 2005; Ritchie \& Touchon, 2000; Shah et al., 2000). To improve the validity of the MCI construct, Petersen et al. (2001) have tried to differentiate various neuropsychological subtypes of MCI, distinguishing "amnestic MCI," from "multiple cognitive domain MCI" and "single nonmemory domain MCI"). In line with this distinction, Petersen et al. (2001) and Dubois and Albert (2004) have shown that the "amnestic" type shows the highest accuracy in predicting progression toward DAT. Furthermore, Petersen et al. (2001) have hypothesized that "multiple domain MCI" is most likely to progress to vascular dementia (VD). However, Lopez et al. (2005) maintained that this classification of MCI patients does not capture the nature of the underlying pathology. Supporting this argument, Nordlund et al. (2005) claimed that very few subjects have isolated memory impair- 
ment and Rasquin et al. (2005) demonstrated that the "multiple domain MCI" subtype is predictive of later development of both DAT and VD.

Some authors have investigated whether MCI patients with different clinical presentations have different patterns of neuropsychological impairment and tend to evolve toward different types of dementia. In particular, these studies attempted to clarify whether MCI patients showing high risk factors for cerebrovascular diseases have a different pattern of neuropsychological impairment from that of MCI patients with no evidence of vascular risk factors. This line of research followed from the observations that, according to some authors, there are differences between the neuropsychological patterns of DAT patients and those of patients with VD resulting from small-vessel disease. The former consistently show a predominance of episodic memory disorders (Gainotti et al., 1989, 2001; Graham et al., 2004; Ingles et al., 2007; Looi \& Sachdev, 1999; Matsuda et al., 1998; Mendez \& AshlaMendez, 1991), whereas the latter appear to show a prevalence of executive dysfunction (Desmond, 2004; Jokinen et al., 2005; Kertesz \& Clydesdale, 1994; Kramer et al., 2002; Looi $\&$ Sachdev, 1999; Padovani et al., 1995). Furthermore, psychomotor retardation, considered to be a prominent consequence of stroke (Ballard et al., 2003; Rasquin et al., 2004) is also found in small-vessel subcortical dementia (Gainotti et al., 2001), in vascular cognitive impairment (Desmond, 2004) and in association with recognized risk factors for vascular dementia, such as white matter hyperintensities (Jokinen et al., 2005). A further difference between DAT and VD could include phonological and semantic fluency, because some authors (e.g., Canning et al., 2004; Jones et al., 2006), but not others (e.g., Bentham et al., 1997; Crossley et al., 1997) have found a greater semantic fluency impairment in DAT patients and a greater phonological fluency impairment in VD patients. On the basis of these findings, it should be logical to expect that different neuropsychological patterns would emerge during the preclinical stages of DAT and VD, respectively. However, the neuropsychological differentiation of DAT from VD is not endorsed by all authors. Almkvist et al. (1993), Meyer et al. (2002) and Laukka et al. (2004) observed, for instance, a similar pattern of cognitive deficits in the preclinical phases of VD and DAT patients. In addition, Reed et al. (2007) recently reported that in patients with autopsydefined DAT and cerebrovascular disease, respectively, executive impairment is not a useful diagnostic marker for VD. These observations suggest that ischemic factors may play an important role in the pathophysiology of both vascular and degenerative dementia (see de la Torre, 2004a,b for recent reviews). This ischemic hypothesis is, however, at variance with several studies, most notably the "Nun study" (Snowdon et al., 1997). This study showed that the presence of several subcortical infarcts does not increase the number of senile plaques or neurofibrillary tangles, suggesting that a chronic brain hypoperfusion is not a main determinant of these lesions.

In this study, we were interested in investigating the predictive accuracy of patterns of neuropsychological impairment, as well as other visual-spatial or episodic memory markers of dementia that have previously demonstrated good accuracy in distinguishing DAT from VD patients (Gainotti et al., 1992) or DAT from other forms of dementia (Gainotti et al., 1998). Therefore, the aim of our study was to evaluate whether different neuropsychological patterns and markers of dementia are apparent in patients with MCI resulting from multiple subcortical infarcts or from hippocampal atrophy, respectively. We advanced the following predictions: MCI patients with hippocampal atrophy (preclinical DAT) should show a predominance of episodic memory disorders, whereas MCI patients with multiple subcortical infarcts resulting from small-vessel disease (preclinical VD) should show significant executive dysfunction and other deficits related to frontal lobe damage, as well as psychomotor retardation. Furthermore, semantic fluency tasks should be more impaired in patients with preclinical DAT (hippocampal atrophy), whereas phonological fluency tasks should be more impaired in patients with preclinical VD (multiple subcortical infarcts). Finally, neuropsychological markers of dementia should be able to differentiate MCI patients with multiple subcortical infarcts from those with hippocampal atrophy.

\section{MATERIAL AND METHODS}

\section{Patients}

Over a 5-year period, approximately 500 patients were referred to our Neuropsychology Service for the onset of a mental deterioration. From this larger patient sample, we selected 104 patients who satisfied MCI criteria (see below) and who could be considered as preclinical DAT or preclinical VD on the basis of the brain MRI.

\section{Criteria used to identify MCI patients}

The clinical criteria suggested by Petersen et al. (1999) were used to identify amnestic MCI patients. These criteria included (1) the presence of subjective memory complaints and objective memory impairment, (2) the absence of impairment in other cognitive domains, and (3) the absence of impairments in activities of daily living. Subjective memory complaints (documenting some form of awareness of the memory deficit) were expected to be confirmed by neuropsychological data. Specifically, at least two scores below cutoff points established for episodic long-term memory tasks (see the neuropsychological battery section) were required to identify objective memory impairment. Other cognitive domains were considered to be impaired only if they had been recognized by patients or by their relatives. Therefore, patients and their relatives were systematically asked whether the patient showed disturbances of language or of judgment, disorders of spatial orientation or difficulties in recognizing objects or persons. The diagnosis of MCI was made only if patients and their caregivers reported an absence of disturbance in these cognitive domains. The absence of functional impairment was systematically doc- 
umented by reviewing ability to perform various daily living activities (ADL).

\section{MRI criteria used to identify MCI patients with multiple subcortical infarcts or with hippocampal atrophy}

All MCI patients underwent an MRI study in which two main criteria, namely: (1) the presence of subcortical brain infarcts less than $2 \mathrm{~cm}$ in size and (2) some degree of hippocampal atrophy, documented with hippocampal volumetry, were taken into account. Hippocampal volumetry was evaluated by means of the segmentation protocol proposed by Pruessner et al. (2000) in a study conducted on 40 normal adults. This method is based on a manual segmentation of the hippocampus and on the use of a three-dimensional software that allows simultaneous analysis of sagittal, coronal and horizontal images. The pulse sequence used in the study was the basic sequence of the Siemens (MPRAGE), with acquisition parameters optimized to obtain a good GM/WM contrast and focused on the hippocampal structure. The images were analyzed in stereotactic space.

Because subjects studied by Pruessner et al. (2000) were younger than our patients, and hippocampal volume decreases with advancing age in healthy adults, we adopted the cutoff points derived by a recent study of Lupien et al. (2007). In this study, several age ranges were considered. The values of $2860 \mathrm{~mm}^{3}$ and $2240 \mathrm{~mm}^{3}$, corresponding respectively to the mean volumetric values minus two standard deviations for the age ranges of 60-75 and 76-85 were taken as cutoff points for the corresponding age groups of MCI patients.

We thought that, because we did not have a control group of normal elderly adults in our study, the most appropriate cutoff points would be $2 S D$ below the mean volumetric values of normal adults of corresponding age identified by Lupien et al. (2007).

On the other hand, MCI patients with subcortical infarcts were identified using a criterion similar to that adopted in a previous personal work on subcortical vascular dementia (Gainotti et al., 2004). MCI patients were included in this group if they presented three or more small subcortical infarcts or two small infarcts and periventricular white matter hyperintensities. The presence of periventricular white matter hyperintensities alone was not considered as a sufficient criterion to be included in the study, even if white matter hyperintensities are often associated with risk factors for vascular disease (Breteler et al., 1994; Longstreth et al., 1996). Patients with both hippocampal atrophy and subcortical infarcts were excluded from the study. On the basis of these strict MRI criteria, $41 \mathrm{MCI}$ patients were included in the group with small subcortical infarcts ("vascular" group), 36 in the group with hippocampal atrophy ("atrophic" group), and 27 were excluded because they showed both vascular and atrophic lesions. A group of 65 normal controls, matched to MCI patients on age and educational level, were also included in the study. Both MCI patients and normal controls were administered the MMSE and an extensive neuropsychological test battery, that can be considered to be an extended version of the Mental Deterioration Battery (Carlesimo et al., 1996). Data included in this manuscript were obtained in compliance with regulations of our ethics review committees, in accordance with the principles outlined in the Helsinki Declaration.

\section{The Neuropsychological Battery}

The neuropsychological battery was based on tests of episodic long-term verbal memory [Rey Auditory Verbal Learning Test (RAVLT): immediate (IR) and delayed recall (DR) and delayed recognition], (Rey, 1958), long-term visualspatial memory [Rey-Osterreith Complex Figure delayed reproduction (ROCFR)] (Lezak, 1995), immediate verbal and visual-spatial memory (Digit and Spatial Span), limb and oral praxis, constructional praxis (Rey-Osterreith Complex Figure copy (ROCFC) and drawing designs with and without landmarks), phonological and semantic/categorical verbal fluency, and the Raven's Standard Progressive Matrices (Raven, 1949), considered as a visual deductive reasoning task. Subjects were also given a group of tests sensitive to frontal lobe lesions, including a demanding attentional task (Multiple Features Targets Cancellation/MFTC) (Gainotti et al., 2001), a test of temporal rule induction (Villa et al., 1990) and the Stroop interference test (Perret, 1974), each of which explores various facets of executive functions. In the MFTC, the patient is presented with an array of 80 small squares, each containing two variously oriented lines, and is asked to cancel as quickly as possible each of the 13 items that match a model placed immediately above the array, whereas in the "Temporal rule induction" the patient is asked to identify and predict the rule according to which binary sequences of red and blue tokens are presented. A further task, aiming to contrast relative impairments of the frontal and temporal lobes consisted of a naming task based both on objects and actions (Miceli et al., 1994). In this task, the patient must name 30 drawings representing high frequency words (cigarette, radio, monkey, etc.) and 28 drawings representing simple actions (to run, to kiss, to cut, etc.), producing nouns and verbs, respectively. Cappa et al. (1998) demonstrated that frontal lobe dementia patients are more impaired naming actions than objects, whereas DAT patients show the opposite pattern of impairment. Similar results, namely a greater impairment in the generation of verbs than in the generation of nouns, have been obtained by Peran et al. $(2003,2004)$ in patients with "subcortical" forms of dementia (Huntington's disease and Parkinson's disease).

Timed tasks, namely phonological $(\mathrm{PhF})$ and categorical verbal (CF) fluency tasks, the lines cancellation (LC) test (Albert, 1973), the Multiple Features Targets Cancellation (MFTC) task and the naming time of the Stroop interference test were used to evaluate psychomotor speed. In addition to the scores obtained on the various tasks of the battery, we also took into account a certain number of neuropsycho- 
logical markers, obtained on the Raven's Matrices, and on drawing designs with and without landmarks and in the RAVLT, that had demonstrated ability to distinguish DAT patients from VD patients and from persons with other forms of dementia (Gainotti et al., 1992, 1998). These markers are (1) the "closing-in" phenomenon on drawing tasks; (2) the tendency to give globalistic or "odd" responses on the Raven's Matrices; (3) the serial position effects (primacy, recency, and recency/primacy ratio) on the immediate recall trials of the RAVLT; (4) the decay of memory traces (absolute memory decay and saving score index) on the Delayed Recall of the RAVLT; (5) the number of false alarms on delayed recognition of the RAVLT. Scores obtained in the various tests and on the putative "neuropsychological markers" of dementia were used to evaluate whether vascular and degenerative forms of MCI can be distinguished on the basis of neuropsychological criteria.

Table 1 reports the main demographic (age, educational level, and gender) characteristics and the mean MMSE values of vascular and atrophic MCI patients and of normal controls.

No difference was found between normal controls and vascular or atrophic MCI patients with respect to demographic characteristics. The MMSE values of MCI patients were, as expected, only mildly impaired (though significantly worse than those obtained by normal controls) and were not significantly different in vascular and atrophic forms of MCI.

\section{Statistics}

Data were analyzed using multiple one-way ANOVAs, with the groups representing the independent variable and the neuropsychological variables constituting the neuropsychological tasks. Post hoc comparisons were carried out by means of multiple "Tukey tests for unequal sample size". Because a large number of variables was analyzed, Bonferroni correction for multiple comparisons was performed and a per-comparison $\alpha$ level of $p=.002$ was considered as significant. Frequency distributions of pathological scores and markers among MCI groups were analyzed by $\chi^{2}$ with Yates correction.

\section{RESULTS}

\section{Patterns of Neuropsychological Impairment}

To evaluate the patterns of neuropsychological impairment shown by atrophic and vascular forms of MCI, we compared mean scores obtained on the neuropsychological tests by normal controls and the two MCI patient categories, and we identified the frequency of pathological performance obtained on these tests by each MCI group.

\section{Mean scores obtained by normal controls and vascular or atrophic MCI patients on the various tests of the Neuropsychological Battery}

Table 2 reports the mean scores obtained by patients with vascular and atrophic forms of MCI and by normal controls on the neuropsychological tests, as well as the time taken to perform the timed tests.

Consistent with the MMSE data, results reported in Table 2 show that the severity of cognitive impairment of our vascular and atrophic MCI patients was quite mild overall, because most test scores were not significantly worse than those obtained by normal controls. As a group, MCI patients were more impaired than controls on episodic memory, naming, and time-dependent attentional tasks. Furthermore, when a comparison was made between atrophic and vascular MCI patients, the former group was strikingly more impaired on episodic memory tasks, and particularly on verbal (RAVLT) and visual-spatial (ROCFR) measures of delayed recall. In addition, atrophic MCI patients were marginally more impaired than the vascular patients with respect to the number of false alarms shown on the MFTC. On the other hand, vascular MCI patients were significantly more impaired only on a test sensitive to left frontal lesions, namely the "Action Naming" task. On some complex timed tests (namely MFCT time and Stroop test - Interference time), both vascular and atrophic MCI patients were slower than normal controls, but not significantly different from each-other. Even if it is possible that different mechanisms could account for the slowing shown by vascular and atrophic forms of MCI, the time taken to perform the MFTC and the Stroop tests did not distinguish vascular from atrophic forms of MCI.

Table 1. Demographic and MMSE comparisons among control subjects and vascular and atrophic MCI

\begin{tabular}{|c|c|c|c|c|}
\hline & $\begin{array}{c}\text { Atrophic MCI } \\
(n: 36)\end{array}$ & $\begin{array}{l}\text { Vascular MCI } \\
\quad(N: 41)\end{array}$ & $\begin{array}{l}\text { Controls } \\
(n: 65)\end{array}$ & \\
\hline & Mean $(S D)$ & Mean $(S D)$ & Mean $(S D)$ & $p$ level \\
\hline Age & $71.20(6.07)$ & $71.65(5.9)$ & $70.98(3.98)$ & .37 \\
\hline Educational level & $9.61(4.8)$ & $8.7(4.44)$ & $9.32(4.12)$ & .66 \\
\hline MMSE & $25.66(2.47)$ & $26.58(1.65)$ & $28.29(1.07)$ & $<.001$ \\
\hline Gender $(\mathrm{M} / \mathrm{F})$ & $18 / 18$ & $26 / 17$ & $37 / 28$ & n.s. \\
\hline
\end{tabular}


Table 2. One-way ANOVA comparisons between vascular and atrophic MCI and Normal controls

\begin{tabular}{|c|c|c|c|c|c|c|}
\hline & \multirow{2}{*}{$\frac{\text { Atrophic MCI }}{\text { Mean }(S D)}$} & & \multirow{2}{*}{$\frac{\text { Vascular MCI }}{\text { Mean }(S D)}$} & & \multirow{2}{*}{$\frac{\text { Controls }}{\text { Mean }(S D)}$} & \multirow[b]{2}{*}{$p$ level* } \\
\hline & & & & & & \\
\hline RAVLT IR. & $25.61(6.1)$ & $=$ & $28.4(7.15)$ & $<$ & $37.29(8.1)$ & .000 \\
\hline RAVLT DR & $1.86(2.49)$ & $<$ & $4.58(2.31)$ & $<$ & $8.26(2.65)$ & .000 \\
\hline RAVLT Recogn.-Hits & $9.97(3.9)$ & $=$ & $10.41(3.33)$ & $<$ & $12.59(1.95)$ & .000 \\
\hline RAVLT Recogn.-False alarms & $5.27(5.53)$ & $=$ & $3.26(5.67)$ & $<$ & $.83(.96)$ & .000 \\
\hline RAVLT Recog.-Accuracy & $74.5(12.01)$ & $=$ & $79.26(16.9)$ & $<$ & $90.61(6.98)$ & .000 \\
\hline ROCFR-recall & $4.17(4.36)$ & $<$ & $9.78(4.71)$ & $=$ & $11.24(8.78)$ & .000 \\
\hline Immediate Visual Memory & $17.62(3.79)$ & $=$ & $18.40(1.75)$ & $=$ & $18.81(2.77)$ & .13 \\
\hline Span-forward & $5.17(0.90)$ & $=$ & $5.05(1.04)$ & $=$ & $5.38(1.28)$ & .31 \\
\hline Span-backward & $3.38(0.86)$ & $=$ & $3.32(0.81)$ & $=$ & $3.92(1.04)$ & .01 \\
\hline LC-hits & $57.7(3.0)$ & $=$ & $59.64(0.54)$ & $=$ & $59.91(.29)$ & .09 \\
\hline LC-time & $62.36(28.7)$ & $=$ & $59.60(37.6)$ & $>$ & $44.65(13.2)$ & .001 \\
\hline MFTC-Hits & $11.074(2.13)$ & $=$ & $10.43(2.50)$ & $<$ & $11.55(1.58)$ & .02 \\
\hline MFTC-False alarms & $2.5(5.7)$ & $>$ & $.87(2.22)$ & $=$ & $.55(.91)$ & .01 \\
\hline MFTC-Accuracy & $90.60(9.08)$ & $=$ & $89.49(9.8)$ & $<$ & $94.0(6.3)$ & .01 \\
\hline MFTC-Time & $102.9(43.9)$ & $=$ & $102.7(46.68)$ & $>$ & $80.12(33.8)$ & .002 \\
\hline Praxis-Limb & $19.1(2.14)$ & $=$ & $19.4(.78)$ & $=$ & $19.5(.84)$ & .07 \\
\hline Praxis-Oral & $18.7(1.98)$ & $=$ & $19.3(1.30)$ & $=$ & $19.2(.67)$ & .03 \\
\hline Praxis-copy & $9.7(2.38)$ & $=$ & $9.1(2.8)$ & $=$ & $9.7(2.9)$ & .53 \\
\hline Praxis-copy with landmarks. & $63.64(12.8)$ & $=$ & $65.3(6.7)$ & $=$ & $64.33(3.5)$ & .28 \\
\hline ROCFR-Copy & $28.08(7.3)$ & $=$ & $27.7(6.99)$ & $<$ & $29.55(4.6)$ & .04 \\
\hline Raven & $23.78(4.04)$ & $=$ & $23.82(3.66)$ & $=$ & $25.2(5.4)$ & .21 \\
\hline Phonological Fluency (PF) & $23.78(8.3)$ & $=$ & $23.2(8.43)$ & $=$ & $24.5(9.6)$ & .63 \\
\hline Categorical Fluency (CF) & $14.41(4.15)$ & $=$ & $14.6(3.87)$ & $=$ & $14.87(3.7)$ & .84 \\
\hline Naming Actions & $26.1(1.98)$ & $>$ & $24.7(3.22)$ & $<$ & $27.1(1.2)$ & .000 \\
\hline Naming Objects & $27.0(2.77)$ & $=$ & $27.4(2.97)$ & $<$ & $29.7(1.3)$ & .000 \\
\hline Stroop test-Interf-T & $59.2(21.56)$ & $=$ & $70.29(26.54)$ & $<$ & $53.7(16.5)$ & .001 \\
\hline Stroop test-Interf-E & $1.95(2.549)$ & $=$ & $1.53(1.77)$ & $=$ & $.90(1.63)$ & .014 \\
\hline
\end{tabular}

*Bold values $p$ correspond to significant differences among groups after Bonferroni correction for multiple comparisons $(p: .05=$ $p: .002)$.

Frequency of pathological performances obtained on the tests of the Neuropsychological Battery by each MCI group

When the number of impaired scores obtained by each MCI group on the tests of the Neuropsychological Battery was considered, results similar to those observed when comparing the means of vascular and atrophic MCI patients were obtained. To demonstrate this comparison, we report the cutoff scores obtained in our control group in Table 3. These cutoff scores, set at $1.67 \mathrm{SD}$ from the control means, corresponded to a statistically derived 5 th percentile of the control group. The number of atrophic and vascular MCI patients scoring below these cutoff points on the various tests of the Neuropsychological Battery are also reported in Table 3.

Data reported in Table 3 show that: (1) on most neuropsychological tasks, the proportion of impaired scores is rather low, both in vascular and in atrophic forms of MCI. (2) The proportion of atrophic MCI patients obtaining an impaired score on the verbal (RAVLT) delayed recall and on the visualspatial (ROCFR) delayed reproduction is, however, very high (approximately 80\%), and a rather high frequency of impaired scores is also observed on the Naming tasks. In particular, vascular MCI patients show a significantly higher number of impaired scores (60.9\%) on the Action Naming task.

In the statistical comparison between atrophic and vascular MCI patients, the former group demonstrated a significantly greater number of impaired scores on the RAVLT DR, RAVLT Recognition-Accuracy and ROCFR recall, and also showed a significantly higher number of false alarms on the MFTC. On the other hand, a significantly higher number of vascular MCI patients obtained impaired scores on the Action naming task.

\section{Neuropsychological Markers of MCI}

Table 4 reports the mean scores (or the frequency of occurrence) of the neuropsychological markers observed in patients with vascular and atrophic forms of MCI. Table 4 also reports the results of the statistical analyses, which were carried out by means of $t$ tests in the comparisons between mean scores and of Yates corrected $\chi^{2}$ in the comparisons between frequencies of occurrence.

Results reported in Table 4 are quite consistent with those observed in Tables 2 and 3 from two points of view: first, only the markers of long-term memory impairment (pri- 
Table 3. Frequency of impaired neuropsychological scores among MCI groups

\begin{tabular}{|c|c|c|c|c|}
\hline & $\begin{array}{l}\text { Cutoff } \\
\text { scores* }\end{array}$ & $\begin{array}{l}\text { Atrophic MCI } \\
\left(N^{\circ}-\%\right)\end{array}$ & $\begin{array}{c}\text { Vascular MCI } \\
\left.N^{\circ}-\%\right)\end{array}$ & $\chi^{2}$ \\
\hline RAVLT IR & $<23$ & $11-30.5$ & $11-26.8$ & .86 \\
\hline RAVLT DR & $<4$ & $30-83.3$ & $11-26.8$ & .000 \\
\hline RAVLT Recogn.-Hits & $<10$ & $9-25.0$ & $11-26.8$ & .93 \\
\hline RAVLT Recogn.-False alarms & $>2$ & $18-50$ & $15-36.6$ & .33 \\
\hline RAVLT Recog.-Accuracy & $<80$ & $21-58.3$ & $15-36.6$ & .09 \\
\hline ROCFR-recall & $<8$ & $28-77.7$ & $19-46.36$ & .01 \\
\hline Immediate Visual Memory & $<14$ & $8-22.2$ & $3-7.3$ & .12 \\
\hline Span-forward & $<4$ & $1-2.7$ & $1-2.4$ & .53 \\
\hline Span-backward & $<3$ & $3-8.3$ & $3-7.3$ & .79 \\
\hline LC-hits & $<59$ & $8-22.2$ & $4-9.75$ & .23 \\
\hline LC-time & $>66$ & $5-13.8$ & $10-24.4$ & .38 \\
\hline MFTC-Hits & $<9$ & $7-19.4$ & $17-41.5$ & .07 \\
\hline MFTC-False alarms & $>2$ & $10-27.7$ & $2-4.8$ & .014 \\
\hline MFTC-Accuracy & $<84$ & $8-22.2$ & $12-29.3$ & .66 \\
\hline MFTC-Time & $>135$ & $7-19.4$ & $11-26.8$ & .62 \\
\hline Praxis-Limb & $<18$ & $1-2.7$ & $1-2.4$ & .53 \\
\hline Praxis-Oral & $<18$ & $4-10$ & $2-4.8$ & .55 \\
\hline Praxis-copy & $<5$ & $2-5.5$ & $5-12.2$ & .53 \\
\hline Praxis-copy with landm. & $<58$ & $5-13.8$ & $5-12.2$ & .90 \\
\hline ROCFR-Copy & $<23$ & $9-25$ & $7-17.1$ & .56 \\
\hline Raven & $<17$ & $2-5.5$ & $1-2.4$ & .90 \\
\hline Phonological Fluency (PF) & $<10$ & $3-8.3$ & $6-14.6$ & .61 \\
\hline Categorical Fluency (CF) & $<9$ & $4-10$ & $2-4.8$ & .55 \\
\hline Naming Actions & $<26$ & $10-27.7$ & $25-60.9$ & .007 \\
\hline Naming Objects & $<28$ & $17-47.2$ & $23-56.1$ & .58 \\
\hline Stroop test-Interf-T & $>80$ & $8-22.2$ & $12-29.3$ & .66 \\
\hline Stroop test-Interf-E & $>3$ & $8-22.2$ & $6-14.6$ & .57 \\
\hline
\end{tabular}

*Cutoff scores were set at $1.67 S D$ from the control means, according to values corresponding to the 5 th percentile of the control groups.

macy effect; primacy minus recency and absolute decay or decay ratio) distinguished the atrophic from the vascular forms of MCI; second, the visual-spatial markers were very rare in both forms of MCI, suggesting that the severity of cognitive impairment was very mild in this sample.
The markers of memory decay on the RAVLT were particularly helpful in differentiating the two groups, because a complete loss of the memory trace in delayed recall was observed in a high percentage $(33 \%)$ of atrophic, but only in very few (2.4\%) vascular forms of MCI.

Table 4. Comparisons of neuropsychological markers among MCI groups

\begin{tabular}{|c|c|c|c|}
\hline & Atrophic MCI & Vascular MCI & \\
\hline Neuropsychological marker & Mean $(S D)$ & Mean $(S D)$ & $p$ level \\
\hline Primacy effect (first 5 words in IR) & $8.30(4.37)$ & $10.26(3.58)$ & .035 \\
\hline Recency effect (last 5 words in IR) & $12.54(4.30)$ & $11.74(4.31)$ & .42 \\
\hline Primacy minus Recency & $-5.12(7.34)$ & $-1.9(6.1)$ & .04 \\
\hline Absolute decay (frequency) & $12 / 36$ & $1 / 41$ & $\begin{array}{c}\mathbf{0 0 0 9} \\
\text { (Yates correct) }\end{array}$ \\
\hline Decay ratio $\frac{[\mathrm{DR}]}{[(\mathrm{IV}+\mathrm{V}) / 2]}$ & $0.29(0.33)$ & $0.68(0.31)$ & .001 \\
\hline Closing In & $1 / 36$ & $0 / 41$ & $\begin{array}{c}.94 \\
\text { (Yates correct) }\end{array}$ \\
\hline Absurde Responses Raven & $1 / 36$ & $0 / 41$ & $\begin{array}{c}.94 \\
\text { (Yates correct) }\end{array}$ \\
\hline
\end{tabular}




\section{DISCUSSION}

Results of our study suggest that a pattern of neuropsychological impairment mainly consisting of episodic memory deficits, is typical of MCI patients with hippocampal atrophy, whereas a different neuropsychological profile, consisting of impaired performance on the Action Naming task is observed in vascular forms of MCI. Two methodological features of the study allow us to maintain that these results are not due to confounding variables, such as the presence of mixed forms of MCI or of a different severity of cognitive impairment in the two matched groups. First, by including in the study only MCI patients whose MRI showed three or more subcortical infarcts, in the absence of hippocampal atrophy ("Vascular MCI") or hippocampal atrophy in the absence of subcortical infarcts ("atrophic MCI"), we excluded all patients with both hippocampal atrophy and subcortical infarcts. Second, we ensured that the severity of cognitive impairment of our vascular and atrophic MCI patients was quite mild overall and that the two groups were well matched in terms of severity of impairment. The generally high MMSE scores and the comparability of mean scores obtained by both MCI groups on most neuropsychological measures confirm that our vascular and atrophic MCI patients were equally mildly impaired.

Returning from the methodological to the factual data, we can say that the severity of episodic memory impairment in MCI patients with hippocampal atrophy was consistently shown: (1) by the very low value of the mean scores obtained by these patients on the on the verbal (RAVLT) and visualspatial (ROCFR) delayed recall tasks of the Neuropsychological Battery, (2) by the very high percentage of patients obtaining impaired scores on the same tasks, and (3) by results obtained when we took into account the neuropsychological markers drawn from the same tests. This pattern of impairment was expected on the basis of the neuropathological concomitants of DAT, because the first elementary lesions in this disease involve the entorhinal cortex and the hippocampus (Braak \& Braak, 1991), disconnecting the Papez circuit from information coming from the outside world and thus hindering the formation of new episodic memories. These data are also consistent with the recent results of van de Pol et al. (2007), who showed that in MCI patients, reduced hippocampal volume at the baseline predicts subsequent accelerated rates of hippocampal atrophy, suggestive of the accumulation of Alzheimer-type pathology, which may clinically manifest itself in the future. This finding does not mean that episodic memory was impaired in isolation in our atrophic MCI patients, because executive functions, naming and performance on timed attentional tasks were also significantly impaired. An explanation of this observation, also framed in terms of the neuropathological staging of DAT, could be that the pathological process was spreading from the temporolimbic to the neocortical association areas in some of our atrophic MCI patients. If this interpretation is correct, during the follow-up of these patients, we should observe that the conversion to DAT should be more rapid and more frequent in patients with severe but not isolated impairment of memory functions. Unfortunately, the number of patients followed up for 1 or more years is, to date, insufficient to check this prediction.

If we pass, now, to the pattern of impairment observed in patients with Vascular MCI, we see that this pattern mainly reflects poor scores on a task sensitive to frontal lobe damage (such as the Action Naming task), but is less salient than that observed in degenerative forms of MCI. This neuropsychological marker seen in our vascular MCI patients, suggesting a likely frontal lobe involvement, is consistent with the hypothesis assuming that small vessel subcortical dementia may be due to the disruption of corticostriatal loops that subserve the functions of the frontal lobes, coursing through the frontal white matter (Alexander \& Crutcher, 1990; Cummings, 1993). The fact that a greater impairment in the generation of verbs than in the generation of nouns has been reported by Peran et al. $(2003,2004)$ in patients with other "subcortical" forms of dementia (Huntington's Disease and Parkinson's Disease) also supports this interpretation.

The greater saliency of the neuropsychological pattern of impairment shown by atrophic MCI patients could be due to three main reasons: (1) the distribution of lesions in vascular MCI patients is rather scattered, being due to disruption of different components of the above mentioned corticostriatal loops, such as the basal ganglia, the thalamus, and the white matter tracts connecting the frontal cortex with these subcortical nuclei (Alexander \& Crutcher, 1990; Cummings, 1993); (2) the nonhomogeneous nature of the cognitive deficits observed in patients with frontal lobe lesions (Dubois et al., 2000; Shallice \& Burgess, 1991); (3) the observation that in patients with nondegenerative forms of MCI, the pattern of cognitive impairment is less consistent and more fluctuating in time. Lopez et al. (2007) have, indeed, rightly noted that when the subject's cognitive deficits could be due to nondegenerative conditions (such as a small-vessel subcortical pathology), a greater proportion of MCI patients remain cognitively stable or return to a normal baseline. These reasons could contribute to explain the conflicting results of previous studies dealing with the patterns of cognitive impairment observed in VD and DAT patients (e.g., Desmond, 2004; Laukka et al., 2004; Looi \& Sachdev, 1999; Meyer et al., 2002; Reed et al., 2007). It is also possible that the absence of a clear neuropsychological pattern in vascular MCI patients was due to their small sample size (and hence to insufficient statistical power ) but this hypothesis is at variance with the fact that a clear and consistent neuropsychological pattern was found in the even smaller group of atrophic MCI patients. In any case, our findings in patients with atrophic and vascular forms of MCI are consistent with results recently obtained by Reed et al. (2007) in DAT and VD patients with autopsydefined pathologies, because both studies show that a severe memory impairment strongly points to an Alzheimer's type brain pathology, whereas the prevalence of executive deficits, other frontal lobe symptoms or psychomotor slowing are a much weaker diagnostic marker of small vessel subcortical cerebrovascular disease. 


\section{ACKNOWLEDGMENTS}

We thank Drs. T. Tartaglione and F. Tomaiolo for their precious contribution in the identification of MCI patients showing multiple subcortical infarcts or a hippocampal atrophy. We also want to express our extreme gratitude to Dr. John L. Woodard for his very careful and time consuming editing assistance, under the auspices of the Research Editing Consultant Program of the International Neuropsychological Society International Liaison Committee. We are very indebted to him, because he improved the clarity and the style of our study.

\section{REFERENCES}

Albert, M.L. (1973). A simple test of visual neglect. Neurology, $23,658-664$.

Alexander, G.E. \& Crutcher, M.D. (1990). Functional architecture of the basal ganglia circuits: Neural substrates of parallel processing. Trends in Neurosciences, 13, 266-271.

Almkvist, O., Backman, L., Basun, H., \& Wahlund, L-O. (1993). Patterns of neuropsychological performance in Alzheimer's disease and vascular dementia. Cortex, 29, 661-673.

Ballard, C., Stephens, S., Kenny, R., Kalaria, R., Tovee, M., \& O'Brien, J. (2003). Profile of neuropsychological deficits in older stroke survivors without dementia. Dementia and Geriatric Cognitive Disorders, 16, 52-56.

Bentham, P.W., Jones, S., \& Hodges, J.R. (1997). A comparison of semantic memory in vascular dementia and dementia of the Alzheimer's type. International Journal of Geriatric Psychiatry, 12, 575-580.

Braak, H. \& Braak, E. (1991). Neuropathological staging of Alzheimer's related changes. Acta Neuropathologica, 82, 239-259.

Breteler, M.M., van Swieten, J.C., Bots, M.L., Grobbee, D.E., Claus, J.J., Vand Den Hout, J.H.W., Van Harskamp, F., Tanghe, H.L.J., De Jong, P.T.V.M., Van Gijn, J., \& Hofman, A. (1994). Cerebral white matter lesions, vascular risk factors, and cognitive function in a population-based study: The Rotterdam study. Neurology, 44, 1246-1252.

Canning, S.J., Leach, L., Stuss, D., Ngo, L., \& Black, S.E. (2004). Diagnostic utility of abbreviated fluency measures in Alzheimer's disease and vascular dementia. Neurology, 62, 556-562.

Cappa, S.F., Binetti, G., Pezzini, A., Padovani, A., Rozzini, L., \& Trabucchi, M. (1998). Object and action naming in Alzheimer's disease and fronto-temporal dementia. Neurology, 50, 351-355.

Carlesimo, G.A., Caltagirone, C., Gainotti, G., \& the Group for the Standardization of the Mental Deterioration Battery. (1996). The Mental Deterioration Battery: Normative data, diagnostic reliability and qualitative analyses of cognitive impairment. European Neurology, 36, 378-384.

Crossley, M., D’Arcy, C., \& Rawson, N.S. (1997). Letter and category fluency in community dwelling Canadian seniors: A comparison of normal participants to those with dementia of the Alzheimer or vascular type. Journal of Clinical and Experimental Neuropsychology, 19, 52-62.

Cummings, J.L. (1993). Frontal-subcortical circuits and human behaviour. Archives of Neurology, 50, 56-67.

de la Torre, J.C. (2004a). Is Alzheimer's disease a neurodegenerative or a vascular disorder? Data, dogma, and dialectics [Review]. Lancet Neurology, 3, 184-190.

de la Torre, J.C. (2004b). Alzheimer's disease is a vasocognopathy: A new term to describe its nature [Review]. Neurological Research, 26, 517-524.
Desmond, D.W. (2004). The neuropsychology of vascular cognitive impairment: Is there a specific cognitive deficit? Journal of Neurological Science, 226, 3-7.

Dubois, B. \& Albert, M.L. (2004). Amnestic MCI or prodromal Alzheimer's disease? Lancet Neurology, 3, 246-248.

Dubois, B., Slachevsky, A., Litvan, I., \& Pillon, B. (2000). The FAB: A frontal assessment battery at bedside. Neurology, 55, 1621-1666.

Gainotti, G., Acciarri, A., Bizzarro, A., Marra, C., Masullo, C., Misciagna, S., Tartaglione, T., Valenza, A., \& Colosimo, C. (2004). The role of brain infarcts and hippocampal atrophy in subcortical ischaemic vascular dementia. Neurological Sciences, 25, 192-197.

Gainotti, G., Marra, C., \& Villa, G. (2001). A double dissociation between accuracy and time of execution on attentional tasks in Alzheimer's disease and multi-infarct dementia. Brain, 124, 731-738.

Gainotti, G., Marra, C., Villa, G., Parlato, V., \& Chiarotti, F. (1998). Sensitivity and specificity of some neuropsychological markers of Alzheimer dementia. Alzheimer Disease and Associated Disorders, 12, 152-162.

Gainotti, G., Monteleone, D., Parlato, V., \& Carlomagno, S. (1989). Verbal memory disorders in Alzheimer's disease and multiinfarct dementia. Journal of Neurolinguistics, 3-4, 327-345.

Gainotti, G., Parlato, V., Monteleone, D., \& Carlomagno, S. (1992). Neuropsychological markers of dementia on visual-spatial tasks: A comparison between Alzheimer's type and vascular forms of dementia. Journal of Clinical and Experimental Neuropsychology, 2, 239-252.

Graham, N.L., Emery, T., \& Hodges, J.R. (2004). Distinctive cognitive profiles in Alzheimer's disease and subcortical vascular dementia. Journal of Neurology, Neurosurgery and Psychiatry, 75, 61-71.

Ingles, J.L., Boulton, D.C., Fisk, J.D., \& Rockwood, K. (2007). Preclinical vascular cognitive impairment and Alzheimer disease: Neuropsychological test performance 5 years before diagnosis. Stroke, 38, 1148-1153.

Jokinen, H., Kalska, H., Mantyla, R., Ylikoski, R., Hietanen, M., Pohjasvaara, T., Kaste, M., \& Erkinjuntti, T. (2005). White matter hyperintensities as a predictor of neuropsychological deficits post-stroke. Journal of Neurology, Neurosurgery and Psychiatry, 76, 1229-1233.

Jones, S., Laukka, E.J., \& Backman, L. (2006). Differential verbal fluency deficits in the preclinical stages of Alzheimer's disease and vascular dementia. Cortex, 42, 347-355.

Kertesz, A. \& Clydesdale, S. (1994). Neuropsychological deficits in vascular dementia vs Alzheimer's disease. Frontal lobe deficits prominent in vascular dementia. Archives of Neurology, $51,1226-1231$.

Kramer, J.H., Reed, B.R., Mungas, D., Weiner, M.W., \& Chui, H.C. (2002). Executive dysfunction in subcortical ischaemic vascular disease. Journal of Neurology, Neurosurgery, and Psychiatry, 72, 217-220.

Laukka, E.J., Jones, S., Small, B.J., Fratiglioni, L., \& Backman, L. (2004). Similar patterns of cognitive deficits in the preclinical phases of vascular dementia and Alzheimer's disease. Journal of the International Neuropsychological Society, 10, 382-391.

Lezak, M.D. (1995). Neuropsychological assessment (3rd ed.). New York: Oxford University Press.

Longstreth, W.T., Manolio, T.A., Arnold, A., Burke, G.L., Bryan, N., Jungreis, C.A., Enright, P.L., O’Leary, D., \& Fried, L. (1996). Clinical correlates of white matter findings on cranial mag- 
netic resonance imaging of 3301 elderly people: The cardiovascular health study. Stroke, 27, 1274-1282.

Looi, J.C. \& Sachdev, P.S. (1999). Differentiation of vascular dementia from AD on neuropsychological tests. Neurology, 53, 670-678.

Lopez, O.L., Becker, J.T., Jagust, W.J., Fitzpatrick, A., Carlson, M.C., DeKosky, S.T., Breitner, J., Lyketos, C.G., Jones, B., Kawas, C., \& Kuller, L.H. (2005). Neuropsychological characteristics of mild cognitive impairment subgroups. Journal of Neurology, Neurosurgery, and Psychiatry, 77, 159-165.

Lopez, O.L., Kuller, L.H., Becker, J.T., Dulberg, C., Sweet, R.A., Gach, H.M., \& DeKosky, S.T. (2007). Incidence of dementia in mild cognitive impairment in the cardiovascular health study cognition study. Archives of Neurology, 64, 416-420.

Lupien, S.J., Evans, A., Lord, C., Miles, J., Pruessner, M., Pike, B., \& Pruessner, J.C. (2007). Hippocampal volume is as variable in young as in older adults: Implications for the notion of hippocampal atrophy in humans. Neuroimage, 34, 479-485.

Matsuda, O., Saito, M., \& Sugishita, M. (1998). Cognitive deficits of mild dementia: A comparison between dementia of the Alzheimer's type and vascular dementia. Psychiatry and Clinical Neurosciences, 52, 87-91.

Mendez, M.F. \& Ashla-Mendez, M. (1991). Differences between multi-infarct dementia and Alzheimer's disease on unstructured neuropsychological tasks. Journal of Clinical and Experimental Neuropsychology, 13, 923-932.

Meyer, J.S., Xu, G., Thornby, J., Chowdhury, M.H., \& Quach, M. (2002). Is mild cognitive impairment prodromal for vascular dementia like Alzheimer's disease? Stroke, 33, 1981-1984.

Miceli, G., Laudanna, A., Burani, C., \& Papasso, R. (1994). Batteria per l'analisi dei deficit afasici (BADA). CEPSAG, UCSC, Roma.

Nordlund, A., Rolstad, S., Hellstrom, P., Sjogrem, L., Hansen, S., \& Wallin, A. (2005). The Goteborg MCI study: Mild cognitive impairment is a heterogeneous condition. Journal of Neurology, Neurosurgery, and Psychiatry, 76, 1485-1490.

Padovani, A., Di Piero, V., Dragoni, M., Iacoboni, M., Gualdi, G.F., \& Lenzi, G.L. (1995). Patterns of neuropsychological impairment in mild dementia: A comparison between Alzheimer's disease and multi-infarct dementia. Acta Neurologica Scandinavica, 92, 433-442.

Peran, P., Démonet, J.F., Pernet, C., \& Cardebat, D. (2004). Verb and noun generation tasks in Huntington's disease. Movement Disorders, 19, 565-571.

Peran, P., Rascol, O., Démonet, J.F., Celsis, P., Nespoulous, J.L., Dubois, B., \& Cardebat, D. (2003). Defisit of verb generation in nondemented patients with Parkinson's disease. Movement Disorders, 18, 150-156.

Perret, E. (1974). The left frontal lobe of man and the suppression of habitual responses in verbal categorical behaviour. Neuropsychologia, 12, 323-330.
Petersen, R.C., Doody, R., Kurz, A., Mohs, R.C., Morris, J.C., Rabins, P.V., Ritchie, K., Rossor, M., Thal, L., \& Winbland, B. (2001). Current concepts in mild cognitive impairment. Archives of Neurology, 58, 1985-1992.

Petersen, R.C., Smith, G.E., Waring, S.C., Ivnik, R.J., Tangalos, E.G., \& Kokmen, E. (1999). Mild cognitive impairment: Clinical characterization and outcome. Archives of Neurology, 56, 303-308.

Pruessner, J.C., Li, L.M., Serles, W., Pruessner, M., Collins, D.L., Kabani, N., Lupien, S., \& Evans, A.C. (2000). Volumetry of hippocampus and amygdala with high-resolution MRI and threedimensional analysis software: Minimizing the discrepancies between laboratories. Cerebral Cortex, 10, 433-442.

Rasquin, S.M., Lodder, J., Ponds, R.W., Winkens, J., Jolles, J., \& Verhey, F.R. (2004). Cognitive functioning after stroke: A oneyear follow-up study. Dementia and Geriatric Cognitive Disorders, 18, 138-144.

Rasquin, S.M., Lodder, J., Visser, P.J., Lousberg, R., \& Verhey, F.R. (2005). Predictive accuracy of MCI subtypes for Alzheimer's disease and vascular dementia in subjects with mild cognitive impairment: A 2-year follow-up study. Dementia and Geriatric Cognitive Disorders, 19, 113-119.

Raven, JC. (1949). Progressive matrices '47, Sets A, Ab, B: Board and book forms. London: Lewis.

Reed, B.R., Mungas, D.M., Kramer, J.H., Ellis, W., Vinters, H.V., Zarow, C., Jagust, W.J., \& Chui, H.C. (2007). Profiles of neuropsychological impairment in autopsy-defined Alzheimer's disease and cerebrovascular disease. Brain, 130, 731-739.

Rey, A. (1958). Mémorisation d'une série de 15 mots en 5 rèpètitions. In A. Rey (Ed.), L'examen clinique en psychologie. Paris: Presses Universitaires de France.

Ritchie, K. \& Touchon, J. (2000). Mild cognitive impairment: Conceptual basis and current nosological status. Lancet, 355, 225-228.

Shah, Y., Tangalos, E.G., \& Petersen, R.C. (2000). Mild cognitive impairment. When is it a precursor to Alzheimer's disease [Review]? Geriatrics, 55, 62, 65-68.

Shallice, T. \& Burgess, P.W. (1991). Deficits in strategy application following frontal lobe damage in man. Brain, 114, 727-741.

Snowdon, D.A., Greiner, L.H., Mortimer, J.A., Riley, K.P., Greiner, P.A., \& Markesbery, W.R. (1997). Brain infarction and the clinical expression of Alzheimer disease. The Nun Study. JAMA, 277, 813-817.

Van de Pol, L.A., van der Flier, W.M., Korf, E.S.C., Fox, N.C., Barkhof, F., \& Scheltens, P. (2007). Baseline predictors of rates of hippocampal atrophy in mild cognitive impairment. Neurology, 69, 1491-1497.

Villa, G., Gainotti, G., De Bonis, C., \& Marra, C. (1990). Double dissociation between temporal and spatial pattern processing in patients with frontal and parietal damage. Cortex, 26, 399407. 